over. The dice were rolled and our Universe was inevitable, somewhere in the Multiverse. And here we are.

Remarkably, we can test this hypothesis. Future experiments will measure the curvature of space with exquisite precision. If the curvature turns out to deviate from flatness, we would come to a conclusion unprecedented in human thought. A slightly closed Universe would prove the finiteness of space. A slightly open Universe would go a long way towards demonstrating that space is infinite, at least in standard cosmologies. If this were the case, we would no longer need to invoke any version of the anthropic principle; it would simply be redundant.

I thoroughly recommend Many Worlds in One. Vilenkin has made some major contributions to the Multiverse hypothesis. Here he illuminates the current issues with clarity and elegance, yet the stories he tells are accessible to non-specialists.

Joseph Silk is in the Department of Physics, University of Oxford, Oxford OX13RH, UK.

\title{
Back on track
}

\section{Out of the Woods: Tales of Resilient Teens by Stuart T. Hauser, Joseph P. Allen \& Eve Golden \\ Harvard University Press: 2006. 327 pp. $\$ 27.95$}

\section{Ann S. Masten}

Stories of individuals overcoming great obstacles to succeed in life no doubt cast their spell on audiences long before they were recorded in books. The power such tales hold will captivate readers of Out of the Woods, an accessible book on resilience by two leading clinical scientists in developmental psychiatry and psychology, Stuart Hauser and Joseph Allen, and independent scholar Eve Golden. Drawing on data from a unique study of 67 teenagers hospitalized for serious behavioural and emotional problems, the authors examine how nine of the troubled teens managed to get 'out of the woods' and on to positive developmental pathways as adults. Their voices emerge from striking interview narratives that span a dozen years, from the time of initial hospitalization (when the future looked bleak indeed) into early adulthood. The achievements and gaps of the resulting book reflect the progress as well as the difficulties of 'resilience science'.

It is intriguing to see a return to case narratives at this particular time in the history of resilience research. Compelling cases often inspired the pioneers who launched the systematic study of human resilience in the 1960s and 70s. Much was learned over the ensuing decades, despite the new field suffering growing pains. A range of diverse studies yielded surprisingly congruent findings about potential protective factors, or 'what makes a difference, although frustratingly few details emerged about how these factors actually work - a situation concisely summarized in the opening chapters.

The heart of the book is focused intensively on the lives of four resilient young people, who are compared and contrasted with each other and with their less adaptive peers. Anyone concerned about young people will find their stories thought provoking. The book reveals the changing voices and perspectives of each individual and shows, in the words of the authors, "how resilience can kick in before it shows". Some of the interview narratives also provide an entertaining and illuminating portrait of unconventional young people thwarting the conventional strategies of their interviewers as the teens struggle to hold their own against family adversity, the indignities of a locked inpatient unit, and the stigma of treatment for mental illness. There are fewer words from the more typical patients; readers must rely on commentary by the authors about the differences.

The case studies emphasize three fundamental protective factors, each a long-standing member of the resilience hall of fame. The first is the presence of positive relationships, encompassing the attachment system and the human capacity for recruiting and forming lasting bonds with parent figures, partners or mentors. The second is agency, the capacity and confidence people have for steering their own lives, and the reward systems that motivate efforts to overcome obstacles and control what is happening in one's life. The third protective factor is reflection on one's self and situation, and the requisite cognitive systems for directing attention and thought. Readers will find other well-established protective factors in these case reports, including optimism about the future and the belief that life has meaning, access to positive institutions, and the advantages of certain cultural or socioeconomic positions.

Largely missing from these vivid accounts, as well as from the first generation of resilience science, is the role of context and multiple levels of analysis. These cases offer a rare and fascinating glimpse into the lives of hospitalized adolescents who have recovered, yet it is difficult to apply the lessons from their lives to other teens and situations. As the authors point out, many details about the developmental histories of these individuals remain unknown. The general role of developmental changes, in person and situation and the way these interact, is not emphasized. Researchers now speculate that the transition to adulthood provides a window of opportunity for late bloomers, when motivation, cognition and opportunity converge to make positive change possible. Brain development enhances the capacity for evaluating, planning and redirecting one's own life. At the same time, young people encounter new and transformational situations, in the form of higher education, job training, military service, religious service or courtship. There is a new wave in resilience science, based on efforts to understand processes across disciplines, levels of analysis and species (humans and animals). Scientists are beginning to integrate recent advances in genomics, neuroscience and the modelling of dynamic systems into the body of knowledge on resilience.

The book serves as a powerful reminder of the phenomenon of resilience and the compelling rationale for understanding resilience well enough to facilitate it. All these young people have suffered, and only $13 \%$ (so far) have made it out of the woods. Their resilience inspires hope, just as individual cases inspired pioneers 40 years ago to hope that science could improve the odds for resilience or prevent children from getting into the woods in the first place. As resilience science matures, the prospects for realizing these goals are improving. Meanwhile, it is important to remember that the woods continue to fill with young people who have lost their way.

Ann Masten is distinguished McKnight university professor of child development, 51 East River Road, Minneapolis, Minnesota 55455-0345, USA.

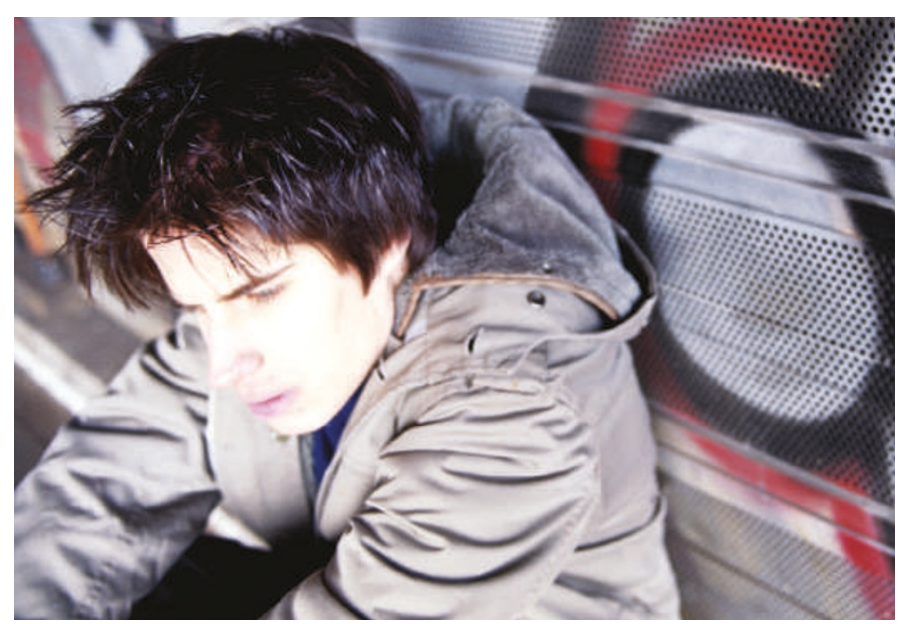

Down but not out: research is progressing on how to help young people overcome their problems. 